\title{
BMJ Open Future Health Today: codesign of an electronic chronic disease quality improvement tool for use in general practice using a service design approach
}

\author{
Barbara Hunter (D) , ${ }^{1}$ Ruby Biezen (D) , ${ }^{1}$ Karyn Alexander, ${ }^{1}$ Natalie Lumsden, ${ }^{1,2}$ \\ Christine Hallinan, ${ }^{1}$ Anna Wood, ${ }^{1}$ Rita McMorrow (D) , ${ }^{1}$ Julia Jones, ${ }^{1,2}$ \\ Craig Nelson, ${ }^{2}$ Jo-Anne Manski-Nankervis (i) ${ }^{1}$
}

To cite: Hunter B, Biezen R, Alexander $\mathrm{K}$, et al. Future Health Today: codesign of an electronic chronic disease quality improvement tool for use in general practice using a service design approach. BMJ Open 2020;10:e040228. doi:10.1136/ bmjopen-2020-040228

- Prepublication history and additional material for this paper is available online. To view these files, please visit the journal online (http://dx.doi.org/10. 1136/bmjopen-2020-040228).

Received 07 May 2020 Revised 17 November 2020 Accepted 19 November 2020

Check for updates

(c) Author(s) (or their employer(s)) 2020. Re-use permitted under CC BY-NC. No commercial re-use. See rights and permissions. Published by BMJ.

${ }^{1}$ Department of General Practice, The University of Melbourne, Melbourne, Victoria, Australia ${ }^{2}$ Western Health Chronic Disease Alliance, Sunshine Hospital, Western Health, Footscray, Victoria, Australia

Correspondence to

Dr Barbara Hunter;

barbara.hunter@unimelb.edu.au

\section{ABSTRACT}

Objective To codesign an electronic chronic disease quality improvement tool for use in general practice. Design Service design employing codesign strategies. Setting General practice.

Participants Seventeen staff (general practitioners, nurses and practice managers) from general practice in metropolitan Melbourne and regional Victoria and five patients from metropolitan Melbourne.

Interventions Codesign sessions with general practice staff, using a service design approach, were conducted to explore key design criteria and functionality of the audit and feedback and clinical decision support tools. Think aloud interviews were conducted in which participants articulated their thoughts of the resulting Future Health Today (FHT) prototype as they used it. One codesign session was held with patients. Using inductive and deductive coding, content and thematic analyses explored the development of a new technological platform and factors influencing implementation of the platform.

Results Participants identified that the prototype needed to work within their existing workflow to facilitate automated patient recall and track patients with or at-risk of specific conditions. It needed to be simple, provide visual snapshots of information and easy access to relevant guidelines and facilitate quality improvement activities. Successful implementation may be supported by: accuracy of the algorithms in FHT and data held in the practice; the platform supporting planned and spontaneous interactions with patients; the ability to hide tools; links to Medicare Benefits Schedule; and prefilled management plans. Participating patients supported the use of the platform in general practice. They suggested that use of the platform demonstrates a high level of patient care and could increase patient confidence in health practitioners.

Conclusion Study participants worked together to design a platform that is clear, simple, accurate and useful and that sits within any given general practice setting. The resulting FHT platform is currently being piloted in general practices and will continue to be refined based on user feedback.

\section{Strengths and limitations of this study}

- Codesign, using a service design approach, was used to inform development of a new chronic disease quality improvement tool.

- General practice staff from regional and metropolitan settings with a broad range of experience in the use of technology participated in the study.

- Iterative technical development process was used to validate codesign principles throughout development.

- General practice and patient participants may not have been representative of these groups more generally.

- Prototype developed through this process requires piloting and further testing to determine fidelity, validity and effectiveness.

\section{BACKGROUND}

More than four in five Australians visit their general practitioner (GP) at least once per year, and 2 million attend each week. ${ }^{12}$ As medical knowledge continues to increase at an exponential rate, it is crucial that this knowledge is translated efficiently and effectively into the general practice setting, where the majority of Australians receive their medical care. This is critically important for people at risk of, or with, three common, interrelated conditions that affect more than 2 million Australians and lead to further health complications, disability and premature death: chronic kidney disease (CKD), cardiovascular disease (CVD) and type 2 diabetes (T2D). ${ }^{3}$ These conditions share risk factors and management strategies which, if put in place early, have the potential to reduce disease progression and the development of complications, improving quality of life and reducing burden on the healthcare system. ${ }^{3}$ As such, there is interest in the 
development and implementation of quality improvement (QI) programmes in general practice targeting these conditions.

Successful QI programmes are multifactorial and can include elements such as audit, feedback and clinical decision support. A Cochrane systematic review of the impact of audit and feedback concluded that potentially important changes in professional practice can be achieved, particularly if feedback is: (1) reported more than once; (2) delivered in multiple formats; and (3) includes explicit targets and action plans. ${ }^{4} \mathrm{~A}$ review of systematic reviews found that changes to professional behaviour are more likely with multifaceted interventions including reminders, audit and feedback that create a set of 'rules' about practice that when enacted become a normal component of everyday practice. ${ }^{5}$ Computerised clinical decision support, combined with other strategies such as the use of key opinion leaders and educational sessions, has the potential to improve health professional performance ${ }^{6}$ and is more likely to be effective if the advice is provided automatically, on the screen, with patient-specific suggestions. ${ }^{78}$ A systematic review and meta-analysis examining the systems of effectively delivering feedback for QI identified development components that were critical for the successful implementation of audit and feedback mechanisms: the method of feedback delivery, the attitude and comprehension of the healthcare professional and the context in which the feedback is delivered all need to align. ${ }^{9}$

Research from Canada and the UK has identified that algorithms developed using data from electronic medical records (EMRs) can accurately identify patients at risk of chronic health conditions in primary care and support QI through audit and feedback. ${ }^{11}{ }^{11}$ These have been delivered to primary healthcare physicians through both paper-based and computerised QI programmes (eg, Performance Improvement plaN GeneratoR: PINGR) and have been tailored to the specific data capture structures (eg, EMR systems used) and health system quirks (including the integration of health services) of the given settings. ${ }^{12-14}$ Challenges associated with implementation of these QI systems include user engagement and ongoing use. Furthermore, successful implementation is influenced by factors such as: ensuring staff QI roles and responsibilities are clearly defined and allocated; the intention and functionality of the initiative are understood and agreed on; the new initiative fits or integrates well with existing systems/protocols; and that sufficient time/resources have been allocated to complete the QI activity. ${ }^{15-23}$ QI systems designed with end users that provide actionable options are most likely to succeed and be sustained over time. ${ }^{24}$

Australian general practices were early adopters of EMRs in the 1990s, with near universal computerisation by $2006 .{ }^{25}$ The data stored within these records can be harnessed to facilitate QI activities and facilitate the translation of research into practice. The Australian government introduced a QI Practice Incentive Payment for general practices in August 2019 (requiring submission of data to Primary Health Networks and participation in QI activities), bringing increased focus on QI activities. ${ }^{26}$ The challenge remains to develop a tool for Australian general practice that provides effective systematic QI functionality to improve guideline concordant care for patients at risk of or diagnosed with chronic disease.

The aim of this study was to codesign with end users an electronic chronic disease QI tool incorporating audit and clinical decision support for use by general practice staff. The tool was not intended to replace existing EMR systems. This paper describes the outcomes of the development process.

\section{METHOD \\ Study design}

The QI tool was developed using service design methodology that promotes user-centred development strategy. ${ }^{27}$ This method involved three codesign engagements with general practice staff, one codesign session with patients and an acceptability and feasibility test of the resulting tool through 'think aloud' sessions.

Service design using codesign is a methodology increasingly used in the development of health services technology. It endeavours to include the end user or primary customer in both the initial and ongoing development of the tool to ensure that what is developed meets consumer needs. ${ }^{28}{ }^{29}$ A strength of the codesign process is that it explicitly aims to develop a process or product in partnership with a variety of end users and then to test or pilot the 'result' further with a wider range of end users. Strategies employed in the codesign process included visualisation and mapping of system gaps, potential tool components and opportunities for system integration, and observation of user interaction with the resulting prototype. ${ }^{28}$

\section{Patient and public involvement}

Patients were recruited at the beginning of the project to provide input in the development and refinement of the QI tool (see 'Recruitment' below). They provided meaningful feedback on the acceptability of the tool for patients and on features specifically related to patient recall, through participation in the codesign focus group.

\section{Recruitment}

General practice staff (GPs, practice nurses and practice managers) were recruited through VicReN, the practicebased research and education network at the Department of General Practice, University of Melbourne. ${ }^{30}$ General practices that are currently participating in the Department's Data for Decisions research programme ${ }^{31}$ were approached to participate as they have an interest in datadriven general practice research and represent a wide range of general practice, in terms of billing structure, location (metropolitan, regional and rural practices) and structure (community health centres and private general 
practice). They were invited to participate via newsletter and email.

Patients were recruited by participating GPs using a direct approach. Interested participants contacted the researchers for further information and an invitation to participate, if they met the inclusion criteria. Inclusion criteria comprised patients with one or more chronic disease, or their carer, who have visited a GP at least three times in the last 2 years. This population was approached as they have experienced recall and management for chronic health conditions in general practice.

All participants gave informed consent to participate.

\section{Data collection}

General practice participants

The codesign methodology consisted of an iterative process where participants discussed the QI systems they use, identified barriers and facilitators to QI in chronic disease management that could be addressed by technology and provided feedback into the tool development (see online supplemental appendix A). In each session, participants were provided with information on the status of the development of the QI tool, called 'Future Health Today' (FHT), and were asked to provide comment and feedback. The clear intention, as provided to participants, was to understand the variety of opinions and perceptions they had regarding each stage of development, not to arrive at consensus. The ideas and improvements were incorporated into the tool, subject to technical requirements. A semistructured interview schedule was used to prompt and guide discussion (see online supplemental appendix B). Meetings were held face to face at the Department of General Practice, University of Melbourne.

\section{The first engagement (initial design)}

Service design methodology, using storyboarding to explore the health services journey, was used to inform development of FHT, using CKD as an exemplar. ${ }^{27}$ Participants were asked to prioritise elements of the prototype for development (including concepts identified by the research and technology teams and by participants themselves) and reality check the platform and proposed components within it.

These sessions provided participants with current statistics on the prevalence of chronic disease (including CKD, CVD and T2D) in Australia and asked participants to apply 'blue sky thinking' to QI for chronic disease management in general practice. They were asked to use CKD to describe and discuss how they currently identified at-risk groups (opportunistic vs planned); what they do once the at-risk groups are identified and how they make this determination; how they identify and manage risk in relation to chronic disease management and in relation to data management; how they manage, enter and store data; how well their current data management systems (including EMR and third party applications) function; if and how they plan and document QI and audit; and if they use or would be interested in benchmarking. Finally, participants were asked about proposed FHT functionality-what they would prefer and what they do not like.

\section{The second engagement (functionality)}

These codesign sessions provided participants with a version of the prototype that incorporated many of the features discussed in session 1, described as a 'dashboard'. They focused on deeper discussion of the design aspects of the prototype and specifically on the preferred functionality and priorities for the designers relating to the dashboard. This session included discussions of categorisation and stratification of clinical information; workshopping appearance and basic functionality; and reflecting on issues and preferences discussed in previous sessions.

\section{The third engagement (refinement)}

These codesign sessions provided participants with the next version of the prototype for discussion and asked them to focus on a clinical decision support component to be primarily used at the 'point of care' in consultation. Changes had been made to the system based on previous discussion and these were reviewed and refined through group discussion.

\section{Zoom videoconference sessions}

Separate Zoom videoconference sessions were held for participants that were either not able to attend the face-toface sessions or who were based in regional Victoria and not able to travel to Melbourne. Two sessions were held: the first focused on initial design and functionality and the second focused on refinement (was held on two separate occasions with different attendees on each occasion).

Sessions were recorded using a digital audio and video recorder, and field notes and sketches were collected for the face-to-face sessions.

\section{Patient participants}

The codesign session with patients focused on the components patients felt were important in a system designed to help identify and manage chronic health conditions from the patient perspective. The group were asked questions about and discussed the process of being recalled, seen and managed by a doctor for a chronic health condition (see online supplemental appendix B). They received a demonstration of the prototype tool and explored patient opinions and acceptance of using technology platforms for healthcare and opinions about active participation in recalls for medical appointments.

The session was recorded using a digital audio and video recorder, and field notes were collected. All audio recordings were transcribed and deidentified for analysis.

\section{Think aloud interviews}

Following the codesign sessions, a working prototype was developed, and a subset of general practice codesign panel members were invited to participate in a "think aloud' session at the Department of General Practice, University of Melbourne, where they talked through 
their use of the tool and made suggestions for improvement prior to development of the final prototype. ${ }^{32}$ They were recorded using a digital video recorder and screen capture technology, and field notes were taken.

\section{Data analysis}

\section{General practice codesign sessions}

The analytical structure applied to this phase of the project involved a two-pronged approach. The first stage of analysis involved a content and descriptive analysis of current processes and preferred technological functionality of a new system for identification and management of CKD. A further content analysis of the field notes and interviews reviewed items arising throughout the codesign process to enable a fidelity check at the end of the development phase and throughout the piloting/refinement process to ensure that the final product both met the end user need and remained faithful to the codesign key design features. Using an inductive approach, codes were generated from the data to identify what was currently being used, what was missing and what could go in the new platform. Data were reviewed and coded by two researchers.

A thematic analysis ${ }^{33}$ was then conducted to examine what codesign participants felt was most important in development and implementation. A combination of inductive coding and deductive coding was used.

\section{Patient codesign session}

A thematic analysis was conducted on the data captured in the patient session, examining key issues arising for participants that may influence the development and implementation of the FHT platform.

All analysis was conducted using NVivo qualitative data analysis software (QSR International Pty Ltd, V.12, 2018).

\section{Think aloud sessions}

The think aloud sessions were analysed using content analysis technique. ${ }^{34}$ As sessions were focused specifically on the functionality of the FHT platform, analysis examined issues that arose during the short 'test run' of the software.

\section{RESULTS}

We aimed to recruit ten participants (four GPs, two practice nurses and two practice managers) to the general practice codesign sessions; however, due to significant interest, 17 people were recruited to participate (eight GPs, five PNs and four PMs), representative of practices across metropolitan Melbourne and regional Victoria. Three face-to-face and three zoom videoconference sessions were conducted, with variable attendance across sessions (see table 1). Six participants attended all three codesign sessions, four attended two sessions and the remaining seven attended a single session (initial design $=6$, functionality $=1$ ). Each face-to-face meeting ran for 85-120 min. Each remote session ran for 40-60 min.

Over the six sessions, participants shifted their focus from the blue skies possibilities of FHT to the practical reality of what the platform was best suited to do, using CKD as an example, and how it filled the gaps left by existing QI systems. The evolving discussions refined the intended purpose of FHT and streamlined the activities that should sit within the FHT platform. Participants were enthusiastic about the possibilities for identifying at-risk patients and for filtering and stratifying large databases of patients into a snapshot review of their health status across chronic conditions. Participants felt that FHT needed to be flexible enough to sit across different visual processing styles, EMR systems (participants used three different systems) and general practice structures.

The variability of attendance across the sessions ensured that the codesign process did not develop a dominant participant dynamic and provided opportunity for participants to challenge and refine concepts over the period of codesign. The semistructured interview structure provided prompts for discussion around the given design components and enabled facilitators to explore issues identified by the research team and those raised by participants. Participants were not asked or encouraged to reach consensus and engaged in respectful discussion with each other, sharing and challenging ideas. Common themes emerged, however, from the multiple discussions.

Key features that participants wanted FHT to include, together with illustrative quotes, are summarised in table 2.

Participants also stressed the importance of ease of use, facilitated through clear and agreed language for any terms and tools used on the platform, clear and easy links between their chosen EMR and FHT and snapshots of information with links to further detail, although the nature of the snapshot was influenced by visual processing preferences.

Table 1 Practitioner participation in codesign sessions

\begin{tabular}{|c|c|c|c|c|c|c|}
\hline \multirow[b]{2}{*}{ Role } & \multirow{2}{*}{$\begin{array}{l}\text { Initial design (1) } \\
\text { F2F }\end{array}$} & \multirow{2}{*}{$\begin{array}{l}\text { Functionality (2) } \\
\text { F2F }\end{array}$} & \multicolumn{2}{|c|}{ Zoom (design and functionality) } & \multicolumn{2}{|c|}{ Refinement (3) } \\
\hline & & & Session 1 & Session 2 & $\mathrm{~F} 2 \mathrm{~F}$ & Zoom \\
\hline GP & 3 & 4 & 4 & 2 & 5 & 1 \\
\hline PM & 3 & 1 & 1 & 0 & 1 & 1 \\
\hline Total & 10 & 6 & 6 & 2 & 7 & 2 \\
\hline
\end{tabular}

F2F, Face to face; GP, general practitioner ; PM, Practice manager; PN, Practice nurse. 
Table 2 Key requested features of FHT, with illustrative quotes

\begin{tabular}{ll}
\hline Key feature & Example quotes \\
\hline Ability to track number of patients at risk of CKD & $\begin{array}{l}\text { '[B]ecause people who are likely to have the highest number of risk factors ... are } \\
\text { the group of patients that we are most likely to be able to do something meaningful } \\
\text { for by knowing who they are and capturing who they are. Especially in clinics with } \\
\text { small numbers of doctors, yet with too many patients, being able to focus on the } \\
\text { patients where we are able to make the most meaningful difference is going to be } \\
\text { really helpful'. (Session 2, GP, zoom, rural and metropolitan) }\end{array}$
\end{tabular}

Automated patient recall

'Reminder and recall systems in practice software is inadequate...people are slipping through'. (Session 2, PN, zoom, rural and metropolitan)

Elements to fit within workflow

'When you're in this you want to be in action mode. You've got your data, you've got your information, you know what you want to do and all of a sudden your clinical decision making says "ok, what is my strategy, which do I do next, when do I do it what do I have to do and what order do I need to do it"'. (Session 3, GP, face to face, metropolitan)

Ability to filter data through a range of lenses

'What's really good about that, it came up in the group discussion, a smaller practice with perhaps less enthusiasm for this, you can actually drill down and get quite small numbers to begin with that allows people to get their feet wet with looking at the key issues and looking at trying to change behaviours or introduce medications, and as you grow in confidence you can start softening your filter and capturing a wider group'. (Session 5, GP, zoom, rural)

Incorporation of QI cycles

Links to information, including national guidelines and patient information
'Could you have a print out so that when you have your monthly meetings you can say this is where we started, this is where we are now and of course this is going to help with QI?' (Session 1, PN, face to face, metropolitan)

'If it has the list of identified things and the list of identified assessment, that's what I would use at a glance. We all know what recommended assessment for CKD is, but when we get down the line to people on the orange or red action plan then definitely, you forget how often to check for ... so having that list pop up quickly rather than clicking through is probably more efficient'. (Session 6, GP, face to face, metropolitan)

\section{Relevant patient pathology results displayed in graphical/visual format to facilitate review}

'... but if you did have BP that was green, ACR which was yellow, and the eGFR was red, and you clicked on it, you would see what the last one was, and a trend came up, it would be really helpful to look at the trend'. (Session 1, GP, face to face, metropolitan)

Ability to focus on conditions relevant to individual practice profiles

'My initial thought to that is, what I think you've got there for general practice is excellent. Because what you are doing is you're identifying one of four groups you can allocate that patient to. I think that behind that there is an opportunity for people with a particular interest to refine their search, such as HIV, but to your bread and butter general practitioner that would be of less importance'. (Session 5, GP, zoom, rural)

Ability to track their own practice's activities over time 'That's the helpful part of it- seeing your own practice change'. (Session 1, PN, face
and potentially to review their activity against that of like to face, metropolitan)
practices (benchmarking)

FHT, Future Health Today; GP, general practitioner; QI, quality improvement.

\section{The prototype}

Following the codesign sessions with general practice staff, a prototype was developed. This prototype comprised a 'dashboard' designed to assist general practices to identify and manage patients with chronic health conditions and to manage QI activities. The 'Dashboard' prototype enabled a global view of patient health status (as it related to CKD) across a general practice. Through an initial navigation page users were able to filter the patient group by one of five designated areas for improvement and further facilitate recall (see box 1 ).

The FHT prototype also included a decision support tool that linked with the patients' EMR at the point of care. This clinical decision support tool is activated when a patient file is opened and where the criteria within the evidence-based algorithms used by the FHT platform are met. The 'pop-up' in the corner of the computer screen advises the GP of the patient's CKD status and recommendations for CKD management. This links to a summary and graphs of the patient's recent blood pressure and pathology relevant to CKD and links directly back to the dashboard, relevant clinical guidelines and resources. From this 'pop-up', the GP can action or defer the recommendations, as appropriate.

\section{Think aloud: prototype testing}

Four participants (two GPs, one practice nurse, one practice manager) from the general practice codesign sessions participated in the 'think aloud' prototype testing of the FHT dashboard. Participants each brought a different perspective to the testing, depending on how they would be using the platform. They each provided detailed 


\section{Box 1 The Future Health Today (FHT) 'dashboard'}

The five chronic kidney disease (CKD) Ql areas as seen on the 'dashboard'

1. Patient has risk factors for CKD and may benefit from a kidney health check.

2. Patient has abnormal pathology results and requires confirmatory testing as they may have CKD.

3. Test results indicate CKD is present, but this is not coded in the electronic medical record as a diagnosis.

4. Patient has diagnosed CKD, and their blood pressure requires optimisation.

5. Patient has diagnosed CKD, and cholesterol medication initiation or management is recommended.

Functions within the FHT 'dashboard'

Generate a list of patients to review through their preferred approach (eg, as they attend a usual appointment, or with a specific recall).

Elect to suspend ('Defer') FHT review for individual patients, either for a given period of time or indefinitely.

Process of 'recall authorisation' to ensure that a patient's usual doctor agrees with and authorises the recall of that patient.

Identify areas where a practice's data capture/management may need improvement.

Links to relevant clinical guidelines and resources.

comments on usability and preferences within the dashboard. The point of care clinical decision support tool was not tested with this group.

Overall, while participants thought FHT looked accessible and provided ample information (both for themselves and for patients), they felt that it was overwhelming and difficult to review and would be challenging for less tech savvy individuals. Many of their concerns were similar to the concerns raised in the general practice codesign sessions and were issues that the technical development team were actively working to improve for the final version for clinical testing. Identified issues surrounded streamlining the dashboard for increased ease of use, simplifying and clarifying language used and provision of clear instruction and training to best use all the features of FHT.

\section{Barriers and enablers to implementation}

Codesign session participants discussed factors that could facilitate or impede the implementation of FHT. Some factors were similarly applicable to any new initiative employed at a practice and have been identified in previous research, including clearly defined roles and responsibilities, an understanding of the intention and functionality of the initiative, good fit or integration with existing systems/protocols and sufficient time/ resources. ${ }^{15-23}$

I think that each person, as we were just talking about, needs to know their role. And they need to be trained in their role and they need to stay within their role. And that will prevent the wrong information getting into the wrong arena. Otherwise you'll end up with the thing going wrong, completely wrong... . (Session 1, GP, face to face, metropolitan)

And don't forget that if it's a ten minute consult and that pops up but it's got nothing to do with what the patient has come in for, then it's just going to be a 'close that'. (Session 1, PN, face to face, metropolitan)

Others could be applied to the implementation of other new technology: the need for the platform to be engaging (and not annoying), intuitive (or familiar), useful and easy to use; the need for the platform to be accurate and free from bugs; and the need to be flexible and allow for some individualisation or adaptation to different contexts.

As with any of these things there will be a need for education and you'll have early adopters and you'll have the laggards. I think just keep it simple and to have as much or as little as you want. (Session 5, GP, zoom, rural)

Factors specific to FHT included: the need for the algorithms sitting within FHT to be accurate; the data drawn from the EMR to be accurate and complete; the ability to use the platform for planned and spontaneous interactions; the ability of the programme to be hidden when not required; the ability to link to the MBS; and interactive links and prefilled tools.

I think you've got things there that prioritise by risk, that allow you to manage your cohort if you want to start small and grow, it's got a feature that allows you to opt the patient out for a period of time, or indefinitely, and discussing there the follow up operation of how you get patients in front of you and do that in a manageable way either me fixing with planned visits to the doctor or support enough that they are coming in before. (Session 5, GP, zoom, rural)

... [A]nd user friendly also, in the respect that when it is done it vanishes, we don't want to see it keep coming up because as you say when people see too many prompts they say I'm not even looking. (Session 1, $\mathrm{PN}$, face to face, metropolitan)

... [C] ut out the things you don't need to see, so we only have the risks that we have automatically identified. (Session 6, GP, face to face, metropolitan)

Perceived barriers to implementation included clear ownership, technological complexity and competing priorities. Perceived enablers to implementation included the familiarity of the system functionality, the flexibility of the tool, the simplicity of the technology and the potential to gain from use of the tool.

Participants identified potential ethical/legal concerns relating to the use of technology to assist with QI activities, including the consequences of identifying a patient as having risk factors but not acting on them, of using autofilled forms (eg, management plans) without sufficient oversight, privacy concerns regarding communication 
methods with patients (eg, email and fax) and appropriate allocation of responsibility and venue for discussion of risk factors and recall. However, participants felt that these risks, primarily surrounding practice management of recall and chronic health discussions with patients, were sufficiently mitigated with strategies currently in place in their own practices.

Participants felt that some contexts were more suited to the implementation of FHT, namely practices with more doctors, with practice nurses and with more time available for patient review and building recall lists. They also felt that FHT could only be used when the patient agenda or need was not urgent or where time was left at the end of a consultation.

Participants self-selected to participate in the project and as such demonstrated an openness to new technology and new ways of managing clinical processes. While they indicated variable technological skill and confidence, they expressed confidence that they would be able to use FHT. For some, the more complex functionalities were accessible because of their similarity to existing programmes. Participants were enthusiastic about the possibilities for clinical performance enhancement provided by FHT, seeing their current ad hoc approaches being strengthened by the platform.

\section{A patient perspective}

The patient codesign group was convened to review the prototype and concept with patients who had attended general practices for chronic health conditions. Five people attended these sessions, with four aged over 60 years and one aged $40-49$ years. Three participants were female and two were male. All lived in metropolitan Melbourne. The session ran for approximately $60 \mathrm{~min}$.

Participants acknowledged that their preferences may be influenced by their age and that younger people may have different preferences. They speculated that younger people may be more connected to their mobile devices and prefer communication that was not as 'personal'. However, participants felt that it was important not to make assumptions about the way people use technology.

Participants were well versed in their own health and had extensive experience attending a GP for their health conditions (conditions including type 1 diabetes, chronic obstructive pulmonary disease and hypertension). All had a continuous relationship with one practice/practitioner (including one participant who had visited the same clinic for 50 years). They had experience with being recalled by their GP for a health issue, but only after visiting or having planned tests done.

Participants were comfortable with the use of computers in face-to-face consultations, had no objections to the inclusion of FHT on the screen and no concerns with the traffic light approach; however, one participant felt strongly that the language used on the clinical decision support at the point of care should be clearer and simpler so that patients would understand exactly what the flag was conveying:
... [W] hy wouldn't you just put chronic kidney disease... why wouldn't you put the whole diagnosis there?... When you see all the abbreviations, which I don't know, it leads to other conversations that then the GP has to say 'this is to do with looking into your kidney function'. Why not just say investigate kidney function? (Female)

Participants in the general practice codesign sessions were adamant that patients would benefit from the provision of graphs to understand how their health indicators were progressing over time and that this method would enable greater conversation about why a given treatment plan or course of action was needed. However, participants were concerned that graphs could be manipulated to exaggerate difference or change and felt that the doctor would tell them if something needed to be addressed.

I know where I'm at. If it's outside the range then we talk about it. If it's not then we don't. So I don't need that. (Male)

The discussion about the inclusion of information or links to guidelines indicated that participants were very happy with their own doctors. Patients believed their own doctors would not need to reference guidelines but conceded that less experienced doctors may benefit from guideline access at the point of care. Patients suggested that they would have greater confidence in a doctor that is not their usual doctor if they accessed the additional information on FHT.

\section{Unresolved challenges}

The complexity of patients' non-clinical characteristics and situations (including homelessness, cultural background, socioeconomic status and age) were discussed in both general practice and patient sessions, both in relation to how best to capture this information and use it in the alerts to practitioners and in relation to how best recall patients for further screening or management. While participants were in agreement that a diverse range of factors influence whether and when a patient will visit their GP, the way they interact with their GP and the complexity of providing care to patients, there was uncertainty as to how a technological platform could identify and incorporate these influencing factors, particularly when many factors are not recorded in the patient's EMR (where FHT will draw its information from) nor are EMR programmes set up to capture the full breadth of information in a systematic and consistent manner. General practice participants also described the complexity of establishing a single technological solution or system for recalling patients to the practice given the diversity of non-clinical characteristics of patients. For example, one participant described the shortcomings of traditional mail-out systems where patients were homeless or between addresses, opting instead for a hybrid phone/ email approach. Others, including patients, described 
the presumed preferences of different age groups in receiving contact from a general practice. The discussions indicated that these issues required additional consideration to determine which features could be embedded in the FHT technology and which would form part of the broader implementation of FHT within a QI framework.

A final issue that was very briefly touched on, but not resolved, was how to display recommendations relating to comorbidity and multimorbidity. The group considered options for a prioritisation process, a time-limited condition specific focus and a broader display of all conditions. These discussions did not resolve with a single solution and indicated to the research and development team that a multipronged approach may be required. Development was to focus on the prototype with a single condition to test if the concept was both possible and useful.

\section{DISCUSSION}

Regular audit and feedback has the potential to increase physician awareness of CKD and improve clinical outcomes for patients. ${ }^{35}$ This awareness, coupled with the experience of members of the research team in CKD (clinical and QI), informed the decision to use CKD as the 'test condition' in the development process. Using this exemplar as a handle to focus their thoughts, codesign participants requested (and codesigned) a system that included features in keeping with this best practice approach to QI, ${ }^{42} 35$ including audit, feedback and clinical decision support, and wanted to see guideline concordant recommendations for care while in consultation. In keeping with previous research, participants identified that the prototype needed to work within their existing workflow to facilitate automated patient recall and track patients with/at-risk of specific conditions. ${ }^{5}$ It needed to be simple, provide visual snapshots of information and easy access to relevant guidelines and facilitate QI activities. This combination of features should work to alleviate the barriers to implementation of guideline concordant care, as identified by Vest $e t$ al and others, including knowledge of the chronic condition, engagement with patients/specialists, time demands and access to/ability to use data. ${ }^{15-23} 36$ The challenge for the FHT technical development team was to operationalise this to find a balance between comprehensive information provision and too much information, between appropriately timed alert and recurrent annoyance, and between succinct and coherent delivery of complex information and over simplification. Evaluation of the implementation of the prototype in multiple general practice settings will provide greater understanding of whether these features are effective in supporting QI.

Codesign has been used effectively in a broad range of healthcare settings to improve physician engagement with QI activities. ${ }^{37} 38$ The inclusion of the 'think aloud' sessions enhanced this codesign process and enabled the developers to test run their concepts, to determine where the design was not complying with the user requirements and to revise the prototype to resolve these concerns.

A key component of successful QI is the level and nature of involvement of the end users, in this case the healthcare professionals. ${ }^{9}$ Those who participated in this project wanted to develop and test the proposed FHT platform and find new ways to improve their responses to chronic healthcare. The process itself generated useful ideas for technological development and reflections on the ways the technology would be used in practice, particularly in conjunction with existing technologies, tools and work practices. Issues and challenges identified by participants were reflective of issues common to the introduction of new technology and new programmes (as discussed briefly in the background section of this report), as were the described facilitators of success.

Participants in the codesign process were drawn from a diverse range of contexts, with varying access to resources, vastly different staffing arrangements, patient lists and capacity for new interventions. The breadth of experience and knowledge contributed by the general practice participants, patients and the research and development team has enriched the design process, enabling the conceptualisation of a flexible platform designed to improve patient health outcomes. Over the codesign journey, it was clear that participants were visualising how they could use FHT in their own daily work to set goals and targets in relation to CKD. In contrast to 'top down' approaches to QI intervention design, this design process enabled the researchers to identify and resolve possible barriers to implementation specific to this particular group of end users before implementing FHT. However, participants may not have been representative of these groups more generally, and broader consultation needs to be undertaken to determine the acceptability and usefulness of FHT to a broader general practice and patient audience.

In recognition of the central role patients play in their own health journeys, ${ }^{39}$ patients were consulted about the acceptability of FHT in primary care. Participating patients also supported the use of the platform in general practice. They felt that use of the platform demonstrated a high level of patient care and could increase patient confidence in health practitioners. Further consultation with patients who have been identified using the FHT platform will provide additional insight on patient experience. Similarly, further piloting and evaluation will provide insight into the usefulness of FHT for QI activities across a range of different general practice settings.

The next step for the FHT project was to pilot the prototype in two different general practice settings and undertake an evaluation of the implementation process (completed in early 2020, results as yet unpublished) using the framework for effective audit and feedback developed by Brown et at ${ }^{\prime}$, Clinical Performance Feedback Intervention Theory. Further refinement and piloting of FHT in additional general practice settings in 2020-2021 will determine the specific impact of contextual factors 
on implementation and ongoing use of FHT and the usefulness and acceptability of the platform to GPs, nurses and practice managers. Further development of the tool is underway to include multiple chronic health conditions (including CKD, CVD, T2D and prostate cancer). A pragmatic cluster randomised control trial is planned to commence in late 2021 to further test the usefulness of FHT in improving outcomes for patients.

\section{CONCLUSION}

The aim of this study was to codesign with end users an electronic QI tool incorporating audit and clinical decision support for use by Australian general practice staff to support chronic disease management. This approach has been a practical and acceptable method for bringing together ideas, concepts and end user needs to develop a platform that can be integrated into the general practice clinical workload. Challenges with QI applications remain an ongoing challenge. However, the resulting FHT version 1 platform is being tested in the general practice pilot sites to determine fidelity to design intentions, acceptability and usefulness of the tool and factors influencing implementation.

To ensure that future development of the FHT platform continues to be informed by real world need an advisory group comprising GPs, practices nurses and practice managers will be established. This group will sit alongside a consumer (people with/who care for people with a chronic condition) advisory group and both will provide advice and guidance on future testing and development of the FHT platform.

\section{Twitter Jo-Anne Manski-Nankervis @jo_manski}

Acknowledgements The authors would like to acknowledge the contributions of the Future Health Today (FHT) project team and investigators. We would also like to acknowledge the time and commitment of all codesign participants.

Contributors J-AM-N, an experienced researcher and academic general practitioner (GP), developed the study design, which was refined following feedback by $\mathrm{RB}$ ( $\mathrm{PhD}$ and mixed methods researcher), NL (PhD and QI researcher) and CN (nephrologist). RB and AW (experienced researcher) undertook recruitment of participants. J-AM-N conducted the codesign sessions with support of RB and JJ (nephrologist) and RB conducted the think aloud interviews. KA was a participant in the codesign sessions. $\mathrm{BH}(\mathrm{PhD}$ and experienced qualitative researcher), and $\mathrm{RM}$ (academic GP and qualitative researcher) performed the analysis of the data. $\mathrm{BH}$ drafted the manuscript with support from $\mathrm{CH}$ ( $\mathrm{PhD}$ and experienced researcher) and $\mathrm{KA}$ (PhD, GP and experienced researcher). All authors revised all drafts and approved the final version of the manuscript.

Funding The FHT project is a multiyear project supported by the Paul Ramsay Foundation (award/grant number: N/A) and the Australian Government's Medical Research Future Fund (MRFF) Rapid Applied Research Translation programme in conjunction with the Melbourne Academic Centre for Health (award/grant number: $\mathrm{N} / \mathrm{A}$ ). The latter fund also provided salary support to Karyn Alexander. J-AM-N is supported by a Next Generation Clinical Researchers Programme - TRIP Fellowship Funded from the MRFF.

Competing interests None declared.

Patient consent for publication Not required.

Ethics approval Ethics project approval was granted by the Melbourne Health Human Research Ethics Committee, The Royal Melbourne Hospital (Ethics ID: HREC/47394/MH-2018) and registered with the University of Melbourne Human Ethics Sub-Committee (Ethics ID: 1852972).
Provenance and peer review Not commissioned; externally peer reviewed.

Data availability statement Deidentified data are available on reasonable request. Deidentified data can be requested from the Research Lead, JMN via the FHT website or contact-FHT@unimelb.edu.au.

Supplemental material This content has been supplied by the author(s). It has not been vetted by BMJ Publishing Group Limited (BMJ) and may not have been peer-reviewed. Any opinions or recommendations discussed are solely those of the author(s) and are not endorsed by BMJ. BMJ disclaims all liability and responsibility arising from any reliance placed on the content. Where the content includes any translated material, BMJ does not warrant the accuracy and reliability of the translations (including but not limited to local regulations, clinical guidelines, terminology, drug names and drug dosages), and is not responsible for any error and/or omissions arising from translation and adaptation or otherwise.

Open access This is an open access article distributed in accordance with the Creative Commons Attribution Non Commercial (CC BY-NC 4.0) license, which permits others to distribute, remix, adapt, build upon this work non-commercially, and license their derivative works on different terms, provided the original work is properly cited, appropriate credit is given, any changes made indicated, and the use is non-commercial. See: http://creativecommons.org/licenses/by-nc/4.0/.

\section{ORCID iDs}

Barbara Hunter http://orcid.org/0000-0002-1268-3166

Ruby Biezen http://orcid.org/0000-0003-1736-9675

Rita McMorrow http://orcid.org/0000-0002-2835-9504

Jo-Anne Manski-Nankervis http://orcid.org/0000-0003-2153-3482

\section{REFERENCES}

1 Hayes P. No one knows you like your GP. newsGP, 2018.

2 Britt H. General practice activity in Australia 2014-15. general practice series No. 38. Sydney: Sydney University Press, 2015.

3 Australian Institute of Health and Welfare (AlHW). Cardiovascular disease, diabetes and chronic kidney disease - Australian facts: Prevalence and incidence, in Cardiovascular, diabetes and chronic kidney disease series no. 2. Canberra: AlHW, 2014.

4 Ivers N, Jamtvedt G, Flottorp S, et al. Audit and feedback: effects on professional practice and healthcare outcomes. Cochrane Database Syst Rev 2012;6:CD000259.

5 Johnson MJ, May CR. Promoting professional behaviour change in healthcare: what interventions work, and why? A theory-led overview of systematic reviews. BMJ Open 2015;5:e008592.

6 Garg AX, Adhikari NKJ, McDonald H, et al. Effects of computerized clinical decision support systems on practitioner performance and patient outcomes: a systematic review. JAMA 2005;293:1223-38.

7 Van de Velde S, Heselmans A, Delvaux N, et al. A systematic review of trials evaluating success factors of interventions with computerised clinical decision support. Implement Sci 2018;13:114.

8 Pefanis A, Botlero R, Langham RG, et al. eMAP:CKD: electronic diagnosis and management assistance to primary care in chronic kidney disease. Nephrology Dialysis Transplantation 2016;98:gfw366:121-8.

9 Brown B, Gude WT, Blakeman T, et al. Clinical performance feedback intervention theory (CP-FIT): a new theory for designing, implementing, and evaluating feedback in health care based on a systematic review and meta-synthesis of qualitative research. Implement Sci 2019;14:40.

10 Brown B, Balatsoukas P, Williams R, et al. Interface design recommendations for computerised clinical audit and feedback: hybrid usability evidence from a research-led system. Int $J$ Med Inform 2016;94:191-206.

11 Tu K, Wang M, Young J, et al. Validity of administrative data for identifying patients who have had a stroke or transient ischemic attack using EMRALD as a reference standard. Can J Cardiol 2013;29:1388-94.

12 Brown B, Balatsoukas P, Williams R, et al. Multi-method laboratory user evaluation of an actionable clinical performance information system: implications for usability and patient safety. J Biomed Inform 2018;77:62-80.

13 Meijers JMM, Halfens RJG, Mijnarends DM, et al. A feedback system to improve the quality of nutritional care. Nutrition 2013;29:1037-41.

14 Dowding D, Randell R, Gardner P, et al. Dashboards for improving patient care: review of the literature. Int J Med Inform 2015;84:87-100.

15 Patel B, Usherwood T, Harris M, et al. What drives adoption of a computerised, multifaceted quality improvement intervention for 
cardiovascular disease management in primary healthcare settings? a mixed methods analysis using normalisation process theory. Implement Sci 2018;13:140.

16 Orchard J, Li J, Gallagher R, et al. Uptake of a primary care atrial fibrillation screening program (AF-SMART): a realist evaluation of implementation in metropolitan and rural general practice. BMC Fam Pract 2019;20:170.

17 Grant A, Dreischulte T, Guthrie B. Process evaluation of the data-driven quality improvement in primary care (DQIP) trial: case study evaluation of adoption and maintenance of a complex intervention to reduce high-risk primary care prescribing. BMJ Open 2017;7:e015281.

18 Litchfield I, Gill P, Avery T, et al. Influences on the adoption of patient safety innovation in primary care: a qualitative exploration of staff perspectives. BMC Fam Pract 2018;19:72.

19 Nouwens E, van Lieshout J, Wensing M. Determinants of impact of a practice accreditation program in primary care: a qualitative study. BMC Fam Pract 2015;16:78.

20 Jeffries M, Phipps DL, Howard RL, et al. Understanding the implementation and adoption of a technological intervention to improve medication safety in primary care: a realist evaluation. BMC Health Serv Res 2017;17:196.

21 Lin IB, Coffin J, O'Sullivan PB. Using theory to improve low back pain care in Australian Aboriginal primary care: a mixed method single cohort pilot study. BMC Fam Pract 2016;17:44.

22 Borg SJ, Crossland L, Risk J, et al. The primary care practice improvement tool (PC-PIT) process for organisational improvement in primary care: application by Australian primary health networks. Aust J Prim Health 2019;25:185-91.

23 Larkins S, Carlisle K, Turner N, et al. 'At the grass roots level it's about sitting down and talking': exploring quality improvement through case studies with high-improving Aboriginal and Torres Strait Islander primary healthcare services. BMJ Open 2019;9:e027568.

24 Foy R, Skrypak M, Alderson S, et al. Revitalising audit and feedback to improve patient care. BMJ 2020;368:m213.

25 Mclnnes DK, Saltman DC, Kidd MR. General practitioners' use of computers for prescribing and electronic health records: results from a national survey. Med J Aust 2006;185:88-91.
26 Department of Health. Practice incentives program quality improvement incentive guidelines. Canberra: Australian Government Department of Health, 2019.

27 Stickdorn M, Schneider J. This is Service Deisgn Thinking: Basics Tools - Cases. Amsterdam: BIS Publishers, 2017.

28 Trischler J, Scott DR. Designing public services: the usefulness of three service design methods for identifying user experiences. Public Management Review 2016;18:718-39.

29 Perrott BE. Including customers in health service design. Health Mark Q 2013;30:114-27.

30 Soós M, Temple-Smith M, Gunn J, et al. Establishing the Victorian primary care practice based research network. Aust Fam Physician 2010;39:857.

31 Boyle D, Emery J, Gunn J, et al. Patron primary care research data Repository. Dataset 2019.

32 Boren T, Ramey J. Thinking aloud: reconciling theory and practice. IEEE Trans Prof Commun 2000;43:261-78.

33 Ritchie J, Lewis J. Qualitative research practice: a guide for social science students and researchers. Thousand Oaks, USA: SAGE Publications Ltd, 2003

34 Erlingsson C, Brysiewicz P. A hands-on guide to doing content analysis. Afr J Emerg Med 2017;7:93-9.

35 Lusignan Sde, de Lusignana S, Gallagher H, et al. Audit-based education lowers systolic blood pressure in chronic kidney disease: the quality improvement in CKD (QICKD) trial results. Kidney Int 2013;84:609-20.

36 Vest BM, York TRM, Sand J, et al. Chronic kidney disease guideline implementation in primary care: a qualitative report from the translate CKD study. J Am Board Fam Med 2015;28:624-31.

37 Zimbudzi E, Lo C, Robinson T, et al. The impact of an integrated diabetes and kidney service on patients, primary and specialist health professionals in Australia: a qualitative study. PLoS One 2019;14:e0219685

38 Brehaut JC, Colquhoun HL, Eva KW, et al. Practice feedback interventions: 15 suggestions for optimizing effectiveness. Ann Intern Med 2016;164:435-41.

39 Domecq JP, Prutsky G, Elraiyah T, et al. Patient engagement in research: a systematic review. BMC Health Serv Res 2014;14:89. 